\title{
LA SATISFACCIÓN CON LA DEMOCRACIA EN EL PERÚ: ANÁLISIS DESDE EL PERFIL DE LOS VOTANTES DE PERUANOS POR EL KAMBIO Y FUERZA POPULAR TRAS EL BALLOTAGE DE LAS ELECCIONES PRESIDENCIALES DE 2016
}

\author{
Satisfaction with democracy in Peru: analysis from the profile of the Peruanos por el Kambio \\ voters' and Fuerza Popular voters' after the second round of the 2016 presidential elections
}

\author{
MARÍA ESTHER MELGAR APAGÜEÑO ${ }^{51}$ \\ Universidad de Santiago de Compostela \\ mariaesther.melgar@,rai.usc.es maria.melgarap@gmail.com
}

Resumen: El siguiente trabajo tiene como finalidad comprender, en general, los factores que influyen en la satisfacción e insatisfacción con la democracia en el caso peruano a la luz de la influencia de los resultados de la segunda vuelta electoral de 2016 -determinación del perfil de los peruanos a través del recuerdo de voto a ambos candidatos. Para el estudio del fenómeno, la investigación ha sido conducida desde la metodología cuantitativa, centrándose en la evidencia empírica cuya base informativa son las percepciones ciudadanas sobre el funcionamiento de las instituciones públicas, las valoraciones de los líderes políticos, la confianza de las instituciones, valoración de la situación económica actual, entre otras que evalúan la calidad del sistema político y las cuales fueron recogidas en la encuesta de opiniónrealizada por el Instituto de Opinión Pública de la Pontificia Universidad Católica del Perú en octubre de 2016.

Palabras clave: satisfacción con la democracia, insatisfacción con la democracia, recuerdo de voto, elecciones generales presidenciales, segunda vuelta electoral.

Abstract: The following work aims to understand, in general, the factors that influence satisfaction and dissatisfaction with democracy in the Peruvian case in light of the influence of the results of the second round of 2016 - determining the profile of Peruvians through the remembrance of vote to both candidates. For the study of the phenomenon, the research has been conducted from the quantitative methodology, focusing on the empirical evidence who sein formative basis is the public perceptions of the functioning of public institutions, the evaluations of political leaders, the trust of institutions, valuation of the current economic situation, among others that evaluate the quality of the political system and which were collected in the opinion pollconducted by the Public Opinion Institute of the Pontificia Universidad Católica del Perú in October 2016.

Keywords: satisfactionwithdemocracy, dissatisfactionwithdemocracy, remembranceof vote, presidential general elections, second round ofelections, politicalparties.

Recibido: 2018-12-04 Aceptado: 2019-10-31. ID: 5651.http://dx.doi.org/10.15304/marco.5.5651

SI. Candidata a doctora en Marketing Político y Actores en las Sociedades Contemporáneas por la Universidad de Santiago de Compostela, Galicia - España. Máster en la Especialidad de Marketing, Consultoría y Comunicación política por la Universidad de Santiago de Compostela, maestra en Ciencia Política y Gobierno por la Pontificia Universidad Católica del Perú y Licenciada en Ciencia Política por la Universidad Nacional Mayor de San Marcos. Consultora y asesora de campañas electorales y analista política en la Agencia peruana BOOM Political Strategies. Docente universitaria de las cátedras de Ciencia Política y tesis de fin de carrera. Investigadora en temas de comunicación política, marketing político, democracia, Mass media; agendación, agendas y agenda-setting; framing, medios y actores políticos, comunicación gubernamental y políticas públicas. Este estudio forma parte del Trabajo Fin de Máster (TFM) dirigido para optar por el grado de máster en Marketing, Consultoría y Comunicación Política. 


\section{INTRODUCCIÓN}

El siguiente trabajo desarrollado aborda el análisis del estado actual de la satisfacción con la democracia en el Perú, teniendo en cuenta la base de la teoría que la sustenta y, asimismo, la data empírica sobre la percepción ciudadana sobre el fenómeno de estudio. Así, la siguiente investigación tiene por finalidad evaluar la calidad de la democracia en el Perú, tras el último proceso electoral de2016.

Para esto, se realizará un análisis descriptivo de la satisfacción con el sistema democrático como tal y según el recuerdo de voto de los ciudadanos como variable dependiente. En este sentido, se realizará un estudio de los factores que inciden en la satisfacción con la democracia, según los resultados electorales obtenidos en las elecciones generales presidenciales de 2016 entre los ganadores y perdedores. -el ballotage entre Peruanos por el Kambio y Fuerza Popular.

La justificación de este trabajo es que este busca determinar los factores que influye en la satisfacción de la democracia, teniendo en cuenta el perfil político de los ciudadanos y en un periodo electoral concreto. Asimismo, en el campo de lo fáctico, este estudio permitirá comprender la problemática que aún se cierne sobre la necesidad de explicar la satisfacción con la democracia en el Perú, los factores que la sustentan y sus efectos en cuanto a la debilidad o estabilidad de los gobiernos, lo cual, eventualmente, afectaría a la estabilidad del propio sistema político en general.

\section{SATISFACCIÓN DEMOCRÁTICA Y EL COMPORTAMIENTOS DEL VOTO: FUNDAMENTOS PARA SU ANÁLISIS EN AMÉRICA LATINA Y EL ESTUDIO DE CASO DEL SISTEMA POLÍTICO PERUANO}

Ciertamente, la democracia es considerada -aunque siempre con sus altas y bajas- hoy como el mejor sistema político que la humanidad ha establecido para asegurar la transferencia del poder de forma periódica, sistemática, legal y con la certeza de que sus resultados son legítimos ante la voluntad popular ${ }^{52}$. Por ello, es importante el estudio sistemático sobre este, tanto en su apoyo como en sufuncionamiento.

En tanto al apoyo del sistema democrático, tenemos la definición churchiliana de esta, en cuanto que este es el mejor sistema político que pueden tener los estados, frente a cualquier otro sistema de gobierno ${ }^{53}$. En este sentido, también es importante entender que lademocracia es un sistema muy importante debido principalmente por cuánto se rige bajo las premisas de la libertad, de los derechos políticos sociales y económicos, y por la preponderancia del Estado de Derecho que asegure la convivencia social, en general, tras un acertado manejo de los asuntos y del Estado.

\$2. desde la perspectiva de Schumpeter en la definición de la democracia moderna -burocrática y sobre la base de una “voluntad general” menos racional y libre que la que postula la teoría clásica. (Held, 1996:207-209).

53. esto respecto a la pregunta: Ahora le voy a leer algunas opiniones sobre el sistema político en el Perú y quiero que usted me indique con cuál de ellas está usted más de acuerdo: a) La democracia es preferible a cualquier otra forma de gobierno, b) A veces un gobierno autoritario o una dictadura puede ser preferible a un gobierno democrático o c) Me da lo mismo un tipo de u otro. 
Ante esto, no es raro que en América Latina este sistema haya sido implementado debido a sus bondades; no obstante, este proceso no fue del todo fácil, incluso, se viene estudiando cómo nuestros sistemas políticos podrían llegar a ser aún mejores. En este sentido, durante la segunda mitad del siglo XX, la región Latinoamérica vivió un proceso de democratización a lo largo de este y que se ha venido consolidando como el mejor sistema de gobierno y que ha sucedido a otros de tipo autoritario e, incluso,dictatoriales.

Ya a finales de la década de 1980, aún en la región latinoamericana hubo países con gobiernos de corte autoritarios, tanto de derechas como de izquierdas, los cuales habían venido alternándose con los gobiernos democráticos en distintos intervalos de tiempo - periodos de 20 años- (Aliester, Cea y Guerrero, 2015: 87). Por tanto, los procesos de democratización que surgieron a finales de 1980 fueron significativos dado que era un periodo de transición luego de los gobiernos de corte autoritario - tales como los vividos en Argentina, Chile, Perú, Bolivia, entre otros ${ }^{54}$.

Dada esta tendencia cíclica de cambio entre los regímenes de corte democrático y los autoritarios y dictatoriales, fue necesario entender por qué la democracia no se lograba consolidar en la región, a pesar de haber tenido experiencias de este tipo. Por ello, muchos autores como Power (citado en Aliester et al., 2015) han realizado estudios sobre qué es lo que sustenta a las democracias en la región y a través de un análisis determinó que el problema radica en el déficit implícito de su legitimidad como sistema de gobierno -referido al apoyo al sistema democrático.

No obstante, a pesar del pronóstico dado por la academia, la región latinoamericana se rige aún bajo el régimen del sistema político democrático. Por ello, los estudios se centraron sobre todo en determinar los factores o condiciones que deberían tener las democracias para que estas se mantengan en el tiempo y con ello logren consolidarse (Alister, Cea y Guerrero, 2015: 87).

En este contexto, muchos de los estudios han sostenido que la estabilidad del sistema democrático en los países latinoamericanos se sustenta sobre todo en variables externas - globalización, sistema económico mundial e instituciones internacionales-, debido a la preponderancia de la economía como eje articulador de las demandas de los organismos internacionales y por la necesidad de tener una mejor reputación a nivel internacional de la que se tuvo en la década de 1980. Asimismo, una vez entendido que el sistema democrático en los países latinoamericanos es el motor que los impulsa en la modernización (Held citado por Alister, Cea y Guerrero, 2015: 7), ahora la premisa clave es saber cuál es la calidad de la democracia que se ha venido construyendo en estos últimos años.

Por ello, la academia ha venido analizando la calidad del sistema democrático a través de ciertos parámetros o dimensiones, las cuales permiten una medición del fenómeno

54. Los periodos van marcados entre las décadas de 1940 y 1950 aproximadamente como periodos de democracia y las década de 1960 y 1970 como periodos de autoritarismo y gobiernos de dictadura sea civil o militar. En tal sentido, para Seligson (2002) la década de 1990 iba a ser un periodo de gobiernos autoritarios en la región, según sus pronósticos dado esta tendencia cíclica de la región. 
en su contexto específico y a través de la percepción de los ciudadanos sobre el sistema y su funcionamiento -en cuanto si apoyan a este y si se sienten satisfechos con la forma en que este funciona-. Así, se han determinado ciertos parámetros, según autores y desarrollos teóricos, tal y como Corbetta y Pérez-Liñan (2001), Altman y Pérez-Liñan (2002), Diamond y Morlino (2005), Mainwaring y Pérez-Liñan (2008), Levine -Molina (2011), entre otras variables adicionales -niveles de corrupción, igualdad socioeconómica, satisfacción ciudadana, decisión electoral y transparencia y rendición de cuentas-.

En este sentido, aquí no vamos a desarrollar ni estudiar una teoría sobre la satisfacción con la democracia en sentido amplio, dado que no es propósito del trabajo la discusión de la teoría en sí misma. Sin embargo, esta investigación tiene como finalidad entender la satisfacción con la democracia en el Perú desde los datos empíricos obtenidos de la última encuesta post-electoral tras la segunda vuelta de las últimas elecciones presidenciales de 2016. Por ello, la metodología empleada en este estudio es la cuantitativa y será abordado a la luz de los parámetros de evaluación de la calidad democrática que señala, en específico, el trabajo de desarrollado por Diamond y Morlino (2005) cuyas dimensiones son (a) Procedimentales -respeto a la ley, rendición de cuentas, participación, competencia (electoral)-, (b) Sustantivas -libertades y derechos (civiles y políticos), e igualdad-, (c) Resultado -apoyo al sistema democrático y satisfacción con el sistema-.

\subsection{La satisfacción con la democracia y su influencia en el comportamiento electoral en el Perú: Una Revisión a la Teoría del Comportamiento delVoto}

No cabe duda que las elecciones son el acontecimiento más importante en toda democracia, debido a que a través de esta se realiza la transferencia de poder y con ello se escoge un nuevo gobierno de forma legítima y legal. Debido a la importancia de este acontecimiento, la Ciencia Política ha intentado estudiar cuáles son los elementos que influyen en el comportamiento del voto de los ciudadanos, con el fin de lograr un entendimiento de este a través de un análisis de identificación de las relaciones causales (Lazo, 2015:54).

En este sentido, el desarrollo teórico del comportamiento electoral se inició en la década de 1940 en los Estados Unidos de América ${ }^{55}$. Los estudios inicialmente fueron realizdos en la Universidad de Colombia y, luego, en la Universidad de Michigan y, debido a ello, estas instituciones y su trabajo teórico constituyen los pilares más importante y significativos de los estudios sobre el comportamiento electoral. No obstante, adicionalmente de estos estudios rectores y pioneros sobre el comportamiento del voto, existe un tercer desarrollo teórico sobre el comportamiento del voto realizado por Anthony Downs, la cual tiene como enfoque teórico a la económica política derivada del enfoque teórico de la elecciónracional.

El primer estudio sobre la materia fue realizado por Lazarsfeld, Berensol y Guated - bajo la influencia de la sociología de Talcott Parsons- en la Universidad de Columbia ${ }^{56}$,

55. Estos estudios se inician como resultado del desarrollo de la teoría conductista, la cual influye a la Ciencia Política y en el campo de estudio del comportamiento electoral.

56. El estudio intitulado “People Choice's" realizado por Paul Lazarfeld -responsable-, BernandBerelson y Hassel Gaudet - colaboradores- fue desarrollado por el Bureau ofApplied Social Research de la Universidad de Columbia. 
los cuales intentaron brindar una explicación sobre cómo los electores definen su voto en una campaña presidencial atendiendo a factores sociales y políticos, y comprendiendo la influencia de estos en el voto -lo que brindó un enfoque sociológico al modelo de estudio. Así, el trabajo concluyó que el nivel socio-económico, los grupos sociales, la pertenencia a grupos religiosos, la percepción social de las agrupaciones y conjuntos sociales influían en la decisión electoral (Lazo, 2015: 54). El modelo de la investigación se desarrolla bajo la perspectiva del grupo social, en cuanto al estudio del entorno más inmediato. Asimismo, el estudio determina que el voto es considerado un mecanismo de afirmación enfatizando la proporcionalidad de los votos que los ciudadanos asignan a un partido enespecífico.

Asimismo, respecto a los estudios realizados en la Universidad de Michigan, estos fueron realizados por Campbell, Converse, Miller y $\operatorname{Stokes}^{57}$ quienes desarrollaron una línea de investigación del comportamiento del voto enfocada, sobre todo, en factores psicológicos como las ideas y las actitudes respecto al sistema político (Sulmont, 2009 y véase tambiénMontesinos, 2007) -esto en cuanto al gobierno, instituciones políticas, partidos políticos, el contexto social y político, identidad política-partidaria, valoración del candidato y los temas de interés, la ideología, entre otros. Así, según el modelo proporcionado, se entiende que el voto es un mecanismo de afirmación de un proceso de largo plazo a través del cual los individuos han ido formando y construyendo su identificación hacia una opción política en particular (Sulmont, 2009: 1). Como resultado de este estudio, se logró demostrar que las actitudes políticas son un factor determinante en decisión para participar en un proceso electoral, enfatizando la influencia de los factores socio-sicológicos y sus características perceptivas asumiendo al ciudadano como un actor con capacidad de influir e intervenir en la política (Lazo,2015:56).

El tercer modelo que estudia el comportamiento electoral es el desarrollado por el Anthony Downs, cuya base es la economía política desde la perspectiva de la elección racional -rationalchoice-, la cual estudia los procesos políticos, y dentro de ellos el electoral, enfatizando en la racionalidad de los individuos al tomar sus decisiones políticas y electorales. Aquí se destaca la frase "renta de utilidad que genera el voto" la cual es la denominación del beneficio político que genera al votar por cierta agrupación política para que logre llegar al poder. Esta retribución se la da el gobierno por ser el ente generador de políticas públicas y de beneficios para la ciudadanía. Por ello, la teoría tiene una base racional sólida que estima que los individuos analizan todos los beneficios posibles a la hora de tomar decisiones. Como se ve, este modelo estima la necesidad que tienen los ciudadanos de analizar cuidadosamente y calcular los beneficios que obtendrían de votar por un partido y no por otro, lo cual resulta ser un ejercicio bastante complejo a la hora de emitir un voto (Lazo, 2015: 56 y Sulmont, 2009:3).

Finalmente, existen modelos más actuales que tratan de explicar y comprender el comportamiento del voto, ejemplo de estos es el "Issuevoting" -teoría desarrollada en la década de 1960- la cual establece que como supuesto central el cual asume que los ciudadanos

57. El estudio realizado se intitula "The American Voter" realizado en el Center for Survey Research de la Universidad de Michigan, cuyo enfoque se centró en el individuo y sus características sicológicas y personales que definen el comportamiento electoral de estos, en el caso de las elecciones presidenciales deıš2. 
determinan su voto hacia algún partido político, enfatizando en dicha decisión sobre todo "los temas" que gira alrededor del mensaje de campaña por que son considerados importantes y de interés (Lazo, 2015: 57).

Asimismo, otra teoría más reciente es la de las redes y su influencia en el comportamiento del voto -iniciada en la década de 1970 en Inglaterra-, pero con un mayor desarrollo de esta en la década de 1990-, la cual tiene vínculos con la teoría de la antropología fenómeno participativo en las sociedades más complejas en comunidades urbanas. Esta plantea que el individuo desarrolla un conjunto de relaciones específicas de apoyo, colaboración e influencia entre un número limitado de actores. Algunos de los principales actores que desarrollan esta teoría son Emmanuel Lazega, Mauricio Gribaudi, entre otros (Montecinos, 2007: 17).

\subsection{El sistema de partidos en el Perú, el problema del voto y la estabilidad del sistema políti- co en su conjunto: caso de las elecciones de segunda vuelta en las Elecciones Generales2016} Respecto al sistema de partidos en el Perú, existe todo un debate en sí, en particular, y en sí de los sistemas de partidos latinoamericanos, en general. Por un lado, en cuanto la teoría clásica de los sistemas electorales, el sistema electoral peruano es un sistema aún en ciernes (Meléndez, 2019), en tanto que no ha logrado superar la crisis o colapso de dicho sistema cuyo inicio es también aún un tema en discusión. Sin embargo, también con el devenir de la política actual, hay otras tesis que indican que hay una recomposición del "sistema partidario" en el país, gracias al análisis de la reciente evidencia empírica que da muestras de ello, a través de las identidades partidarias (Meléndez, 2019).

Sobre el punto de cuando se inició el quebrantamiento del sistema de partidos, son muchas las teorías que tratan de esbozar el momento exacto en el cual el sistema peruano de partidos colapsó. Ciertas posturas identifican este momento con el episodio histórico de la irrupción de las dictaduras militares en América Latina, en las décadas de sesentas y setentas hasta inicios de 1980, las cuales trajeron a bajo no solo es sistema de partidos sino al mismo sistema democrático. El Perú no fue ajeno a este momento histórico de la región -1968 es el año cuando se da el golpe militar al primer gobierno de Fernando Belaunde Terry, el cual fue el del gobierno revolucionario de las Fuerzas Armadas liderado por General Juan Velazco Alvarado.

Por otro lado, otros autores coincidieron en que la crisis institucional de los partidos en el Perú se reflejó claramente en la época de la transición a la democracia -los gobierno de Fernando Belaunde (1980-1985) y el primer gobierno de Alan García (1985-1990), en los cuales la débil institucionalidad de los partidos políticos se plasmó en la gestión y administración de lo público, lo cual tuvo como resultado el declive económico del país y la anomia institucional y política (inflación y recesión económica, el conflicto armado interno y el desprestigio internacional por el problema del no pago de ladeuda).

No obstante, hay otras posturas que indican que el momento clave para el declive del sistema de partidos fue durante el autogolpe de Estado de Alberto Fujimori, en el cual las fuerzas políticas en el Congreso no tuvieron el poder suficiente para lograr una correlación 
de fuerzas necesarias para la resistencia. Esta última postura la podemos observar en los trabajos de Pease (2008), donde señala que la crisis del sistema de partidos en el Perú se plasmó en las elecciones generales presidenciales y congresales de 1990 y de 1995. La segunda postulación de Alberto Fujimori fue controversial, luego de la interpretación constitucional de 1995 -la cual consideraba como primera postulación dado que esta se regiría en ese momento a lo establecido en el nuevo documento constitucional de 1993 y la que fue denominada como "Interpretación auténtica de la Constitución de 1993". (p. 10)

Aquí, Pease reflexiona que la debilidad del sistema de partidos se tradujo en la amplia aceptación de la postulación de Alberto Fujimori a esas elecciones en contraposición de la impopularidad de los líderes de la oposición rivales políticos de Fujimori, debido a que estos representaban a partidos políticos tradicionales - al establisment-, los cuales ya no contaban con la simpatía de la ciudadanía ni se identificaban con ellos ni a la clase política tradicional en general -crisis de representación democrática de los partidos tradicionales y la desaprobación valorativa de sus líderes.

Este punto de vista es compartido por Meléndez (2012) el cual determina que la crisis del sistema de partidos en el Perú se sitúa en la década de 1990. No obstante, la postura del autor refiere que este proceso tuvo como punto álgido las alecciones del año 2000, luego del cual devino el actual escenario -hasta aproximadamente hasta el 2011- periodo denominado "escenario de post-colapso partidario" (2012: 3-4). Así, Meléndez ensaya una tesis contraria al "sentido común" determinada por la literatura actual tradicional, la cual señalaba que el Perú se encontraba en un periodo en el cual la política carece de un sistema de partidos en sí mismo, dado que hay evidencia de una gran volatilidad del voto, la existencia de una amplia fragmentación y atomización política (Levitsky y Cameron citado por Meléndez, 2012: 3).

Asimismo, su tesis también contradice los argumentos de Sánchez (2009), quien suscribe que es posible que en algunos sistemas democráticos con partidos políticos, no necesariamente se haya conformado en un sistema propiamente dicho, dado que muchas veces estas son organizaciones que trabajan aisladamente - como constelaciones, a pesar de cumplir con los requisitos legales para su existencia, pero que no lograr articularse dentro de un espacio que permita una real competencia (Sánchez citado por Meléndez, 2012:3).

En este sentido, la tesis de Meléndez es que en el Perú -y a pesar de este escenario partidario aún en discusión por la teoría tradicional- existe un proceso de reinstitucionalización partidaria, la cual se demuestra por la evidencia empírica respecto a los siguientes elementos de análisis: la disminución de la volatilidad electoral, la existencia de vínculos políticos predominantemente ideológicos a pesar del personalismo político, el sistema político en su conjunto no se ha debilitado (en cuanto a su legitimidad), y por la configuración de identidades políticas en actores que, en otro momento, eran considerados anti-sistema -o cuyo discurso en un inicio era anti-sistema para su legitimación en su irrupción en la política (Meléndez, 2012 y Meléndez,2019). 


\section{RESULTADOS}

\subsection{Análisis de los determinantes de la satisfacción con la democracia tras las elecciones generales presidenciales 2016 , según el recuerdo de voto de los encuestados respecto a la segunda vuelta electoral}

Modelo 1: Regresión logística sobre la satisfacción con la democracia para los que recuerdan haber votado a Peruanos por el Kambio (PPK) en la segunda vuelta electoral -ballotage- y su candidato Pedro Pablo Kuczynski (losganadores)

El siguiente modelo trabajado intentó encontrar la relación causal entre la satisfacción con la democracia -variable dependiente-, el recuerdo de voto en segunda vuelta -ballotage- al partido político Peruanos por el Kambio (PPK) y su candidato Pedro Pablo Kuczynski como variable filtro o interviniente- y una serie de variables-factores que la determinan tales como la edad, situación laboral, recuerdo de voto en primera vuelta, entre otras -variables independientes. Para ello, los datos fueron sometidos a una rigurosa limpieza antes de su análisis estadístico tales como la búsqueda de los datos perdidos, la evaluación de cada una para determinar su importancia para el modelo, según la literatura que se utilizó como base alrespecto.

A continuación, se observarán los resultados obtenidos del modelo respecto a la variable dependiente "satisfacción con la democracia" y las diversas variables independientes escogidas por este como significativas para su explicación en la tabla siguiente:

Tabla 1. Regresión logística de la satisfacción con la democracia de los que recuerdan haber votado por Pedro Pablo Kuczynski en la segunda vuelta de las Elecciones Generales Presidenciales del 2016

\begin{tabular}{|l|c|}
\hline Variables de estudio & $\begin{array}{c}\text { Regresión logística - satisfacción con } \\
\text { la democracia de los votantes de PPK }\end{array}$ \\
\hline Situación laboral & \multicolumn{1}{|c|}{$0,214^{* *}(1,239)$} \\
\hline $\begin{array}{l}\text { Grado de confianza en la Policía Nacional } \\
\text { (PNP) }\end{array}$ & \multicolumn{1}{|c|}{$0,476)^{*}$} \\
\hline $\begin{array}{l}\text { Grado de confianza en el Jurado Nacional de } \\
\text { Elecciones }\end{array}$ &, $587^{* *}$ \\
\hline $\begin{array}{l}\text { (JNE) } \\
\text { Valoración de la situación económica actual } \\
\text { general }\end{array}$ & \multicolumn{1}{|c|}{, $516^{*}$} \\
\hline $\begin{array}{l}\text { Valoración de la situación económica personal } \\
\text { y familiar en retrospectiva }\end{array}$ & $(1,798)$ \\
\hline
\end{tabular}




\begin{tabular}{|c|c|}
\hline $\begin{array}{l}\text { Aprobación de la gestión de Ministro de } \\
\text { Economía y Finanzas Alfredo Thorne }\end{array}$ & $\begin{array}{l}0,957 * * \\
(2,603)\end{array}$ \\
\hline $\begin{array}{l}\text { Aprobación de la gestión de Verónica Mendoza } \\
\text { en la oposición }\end{array}$ & $\begin{array}{l}-, 882 * * \\
(0,414)\end{array}$ \\
\hline $\begin{array}{l}\text { Aprobación de la gestión de Keiko Fujimori en } \\
\text { la oposición }\end{array}$ & $\begin{array}{l}-0,891 * \\
(0,410) \\
\end{array}$ \\
\hline Auto-ubicación étnica de origen quechua & $-1,356 *(0,258)$ \\
\hline $\begin{array}{l}\text { Los ingresos alcanzan justo y no permiten el } \\
\text { ahorro }\end{array}$ & $0,855^{*}(2,351)$ \\
\hline $\begin{array}{l}\text { Valoración a Keiko Fujimori como lideresa } \\
\text { política }\end{array}$ & $0,159 *(1,172)$ \\
\hline $\begin{array}{l}\text { Valoración a Verónica Mendoza como lideresa } \\
\text { política }\end{array}$ & $0,135^{*}(1,144)$ \\
\hline $\begin{array}{l}\text { Grado de acuerdo sobre la complejidad de la } \\
\text { política y su entendimiento por los ciudadanos }\end{array}$ & $\begin{array}{l}-, 371 * * \\
(0,690)\end{array}$ \\
\hline Constante & $-6,352 * * *(0,002)$ \\
\hline $\mathbf{R}^{2}$ de Nagelkerke & 0,450 \\
\hline$-2 \log$ & 270,202 \\
\hline \multicolumn{2}{|c|}{ El grado de significación se establece en base $\mathrm{a} * * * \mathrm{p}<(0,01) ;{ }^{* *} \mathrm{p}<(0,05) ;{ }^{*} \mathrm{p}<(0,1)$} \\
\hline Fuente: Elaboración propia. Datos obtenidos & OP - PUCP Octubre 2016. \\
\hline
\end{tabular}

Tras el análisis de los resultados podemos decir que la satisfacción con la democracia en el caso peruano tiene como variables explicativas a la situación laboral, grado de confianza en la Policía Nacional, grado de confianza en el Jurado Nacional de Elecciones (JNE), valoración de la situación económica actual del país, valoración de la situación económica personal y familiar en retrospectiva, la aprobación de la gestión del Ministro de Economía y Finanzas Alfredo Thorne, la aprobación de la gestión de Verónica Mendoza en la oposición, la aprobación de la gestión de Keiko Fujimori en la oposición, la auto-ubicación étnica como quechua, respuesta sobre el ingreso, su alcance y la capacidad de ahorro de los ciudadanos; la valoración a Keiko Fujimori como lideresa, valoración a Verónica Mendoza como lideresa política, grado de acuerdo sobre la complejidad de la política y su entendimiento por los ciudadanos.

Asimismo, sobre la bondad de ajuste del modelo, los resultados - en función de las variables incluidas -demuestran y concluyen que éste queda explicado en un 0,450 en el $\mathrm{R}^{2}$ de Nagelkerke; es decir, con las variables seleccionadas e incluidas para el análisis, se puede explicar la satisfacción con la democracia de los peruanos en un $45 \%$ y clasifica correctamente el 75,5\% de los casos, lo que nos permite tener una potente herramienta para identificar los factores sobre la cual se basa y, por lo tanto, se acepta el modelotrabajado. 
Modelo 2: Regresión logística de la satisfacción con la democracia de los ciudadanos que recuerdan haber votado a Fuerza Popular (FP) en segunda vuelta - ballotage- y su candidata Keiko Fujimori (los perdedores).

El siguiente modelo trabajado intentó encontrar la relacional causal entre las la satisfacción con la democracia -variable dependiente-, el recuerdo de voto en segunda la vuelta - ballotage- al partido político Fuerza (FP) y su candidata Keiko Fujimori -como variable filtro o interviniente- y una serie de variables-factores que la determinan tales como la edad, situación laboral, recuerdo de voto en primera vuelta, entre otras -variables independientes.

A continuación, se observarán los resultados obtenidos del modelo trabajado respecto a la variable dependiente "satisfacción con la democracia" y las diversas variables independientes escogidas por este como significativas para su explicación en la tabla siguiente:

Tabla 2. Regresión logística de la satisfacción con la democracia de los que recuerdan haber votado por Keiko Fujimori en la segunda vuelta de las Elecciones Generales Presidenciales del 2016

\begin{tabular}{|l|c|}
\hline Variables de estudio & $\begin{array}{l}\text { Regresión logística de la satisfacción con la de- } \\
\text { mocracia de los votantes de FP }\end{array}$ \\
\hline Sexo & $1,809^{*}(6,107)$ \\
\hline Situación laboral & $0,560^{*}(1,751)$ \\
\hline $\begin{array}{l}\text { Grado de confianza en el Gobierno } \\
\text { Nacional }\end{array}$ & $1,604 * *(4,975)$ \\
\hline $\begin{array}{l}\text { Grado de confianza en el Congreso } \\
\text { de la República }\end{array}$ & $-1,420^{*}(0,242)$ \\
\hline $\begin{array}{l}\text { Grado de confianza en el Jurado } \\
\text { Nacional de Lecciones }\end{array}$ & \\
\hline \begin{tabular}{l} 
(JNE) \\
\hline $\begin{array}{l}\text { Grado de Confianza en la Defen- } \\
\text { soría del Pueblo }\end{array}$
\end{tabular} & $-1,281^{* *}(0,278)$ \\
\hline $\begin{array}{l}\text { Valoración de la situación económi- } \\
\text { ca actual del país }\end{array}$ & $2,033^{* *}(7,634)$ \\
\hline $\begin{array}{l}\text { Percepción de los políticos implica- } \\
\text { dos en actos de corrupción }\end{array}$ & \\
\hline
\end{tabular}




\begin{tabular}{|c|c|}
\hline $\begin{array}{l}\text { Percepción de los funcionarios públicos implicados en actos de } \\
\text { corrupción }\end{array}$ & $-1,769 * *(0,170)$ \\
\hline $\begin{array}{l}\text { Aprobación de la gestión del Presidente del Consejo de Ministros } \\
\text { Fernando Zavala }\end{array}$ & $2,072 *(7,937) *$ \\
\hline Aprobación de la gestión de Verónica Mendoza en la oposición & $-3,643 * *(0,026)$ \\
\hline Respeto al Estado de Derecho & $2,249 * *(9,482)$ \\
\hline Apoyo al sistema democrático & $\begin{array}{l}4,4777 * * \\
(118,741)\end{array}$ \\
\hline Valoración a Pedro Pablo Kuczynski como líder político & $-0,598 * *(0,550)$ \\
\hline Valoración a Verónica Mendoza como lideresa política & $0,362 *(1,436)$ \\
\hline $\begin{array}{l}\text { Grado de acuerdo sobre el entendimiento de la política por los } \\
\text { ciudadanos }\end{array}$ & $-1,168^{*}(0,311)^{*}$ \\
\hline $\begin{array}{l}\text { Grado de acuerdo sobre la preocupación de los políticos en por las } \\
\text { demandas de la ciudadanía }\end{array}$ & $-1,188 * *(0,305)$ \\
\hline Constante & $-8,132 *(0,000)$ \\
\hline $\mathbf{R}^{2}$ de Nagelkerke & 0,727 \\
\hline$-2 \log$ & 82,125 \\
\hline \multicolumn{2}{|c|}{$\begin{array}{l}\text { El grado de significación se establece en base a *** } \mathrm{p}<(0,01) ; * * \mathrm{p}<(0,05) ; * \mathrm{p}< \\
(0,1) .\end{array}$} \\
\hline Fuente: Elaboración propia. Datos obtenidos de IOP - PUCP Octu & 2016. \\
\hline
\end{tabular}

Tras el análisis de los resultados podemos decir que la satisfacción con la democracia en el caso de los que recuerdan haber votado por Fuerza Popular tiene como variables explicativas de su voto al sexo, la situación laboral, grado de confianza en el Gobierno Nacional, grado de confianza en el Congreso de la República, grado de confianza en el Jurado Nacional de Elecciones (JNE), grado de confianza en la Defensoría del Pueblo, valoración de la situación económica actual del país, percepción sobre los políticos implicados en actos de corrupción, percepción sobre los funcionarios públicos implicados en actos de corrupción, la aprobación de la gestión del Presidente del Consejo de Ministros Fernando Zavala, la aprobación de la gestión de Verónica Mendoza en la oposición, respeto al Estado de Derecho, apoyo al sistema democrático, la valoración a Pedro Pablo Kuczynski como líder político, valoración a Verónica Mendoza como lideresa política, grado de acuerdo sobre el entendimiento de la política por los ciudadanos y la preocupación de los políticos por las demandas de laciudadanía. 
Para concluir, respecto a la bondad de ajuste del modelo, los resultados - en función de las variables incluidas - demuestran y concluyen que éste queda explicado en un 0,727 en el $\mathrm{R}^{2}$ de Nagelkerke; es decir, con las variables seleccionadas e incluidas para el análisis, se puede explicar la satisfacción con la democracia de los que recuerdan haber votado por Fuerza Popular y su candidata Keiko Fujimori en un 73\% y clasifica correctamente el 89,5\% de los casos, lo que nos permite tener una potente herramienta para identificar los factores sobre la cual se basa esta y, por lo tanto, se acepta el modelo trabajado.

\section{DISCUSIÓN}

1.1 Factores que influyen en la satisfacción con la democracia para los votantes de Peruanos por el Kambio (PPK) y su candidato Pedro Pablo Kuczynski (los ganadores)

El siguiente modelo trabajado intentó encontrar la relación causal entre la satisfacción con la democracia de los ciudadanos - variable dependiente- que recuerdan haber votado en segunda vuelta - ballotage- por el partido político Peruanos por el Kambio (PPK) y su candidato Pedro Pablo Kuczynski -filtro- y los factores que la determinan o la influyen- variables independientes.

De este modo, de los resultados arrojados por el modelo trabajado se colige que la satisfacción con la democracia de los que recuerdan haber votado por la agrupación política Peruanos por el Kambio tiene como variable explicativa más importante a la aprobación de la gestión del Ministro de Economía y Finanzas del momento, el señor Alfredo Thorne, lo cual determina que mientras más sea aprobada la gestión de este funcionario es más probable que los votantes de PPK se sientan satisfechos con el sistema políticodemocrático.

Asimismo dentro de las variables sociodemográficas, las únicas que acoge el modelo son la de la situación laboral y el nivel de ingresos, las cuales se relaciona directamente con la sensación de satisfacción con el sistema político de los votantes de Peruanos por el Kambio. Así, esto significa que cuanto mayor sea la percepción de estar en una situación laboral la cual me permita, como mínimo, tener ingresos que satisfagan mis necesidades aunque quizá no logre ahorrar-, habrá una mayor satisfacción con la democracia -en este caso el grupo mayoritario de los encuestados respondieron ser independientes o emprendedores.

Respecto a la confianza en las instituciones del estado, el sistema determina como significativas dos variables: la Policía Nacional y el Jurado Nacional de Elecciones (JNE), de lo que se puede interpretar que, para el modelo dado, a mayor confianza tengan los votantes de PPK sobre estas instituciones, mayor será su satisfacción con el sistema democrático en términos de funcionalidad, la Policía Nacional es la encargada de salvaguardar el orden interno y quien lucha contra la inseguridad ciudadana, mientras que el Jurado Nacional de Elecciones es la autoridad máxima jurisdiccional en lo referido a procesos electoral y quien determina las reglas del juego de este, en primera y última instancia.

Respecto a las variables estructurales relevantes para el modelo establecido, se evidencia una relación directa entre la valoración de la situación económica actual del país y la valoración de la situación económica personal y familiar en comparación a la que se tenía 
hace 12 meses, lo cual indica que mientras mayor se valore la situación económica actual, y en retrospectiva personal y familiar con referencia a la situación actual, mayor será la satisfacción que tenga con el sistema democráticos los votantes de PPK.

En cuanto a la aprobación de los principales funcionarios públicos del estado peruano y de la oposición, la variable más relevante y única fue la aprobación de la gestión del Ministro de Economía y Finanzas Alfredo Thorne, y se entiende que mientras más es aprobada la gestión de este -mientras mejor sea su desempeño- mayor será la sensación de satisfacción con el sistema de los votantes de PPK. Además, respecto a la aprobación de la gestión de los líderes de oposición en el gobierno, aquí las variables más significativas son la aprobación de la gestión de Verónica Mendoza y la aprobación de la gestión de Keiko Fujimori en la oposición -ambas en relación inversa-, y esta se relaciona con la variable de estudio en tanto que mientras menos se aprueba la gestión de ellas en la oposición en el gobierno de Kuczynski, mayor será la satisfacción con la democracia en el país para sus votantes.

Otra variable significativa para el modelo de estudio, es la auto-ubicación étnica dentro del grupo de los quechuas pero en sentido inverso, con lo cual podría entenderse que mientras menos sean los votantes de PPK de este grupo étnico - o se auto-ubiquen-, mayor será la satisfacción con el sistema democrático. La interpretación en este caso podría darse dada la existencia de una marcada diferenciación entre los grupos étnicos en el Perú y su adecuado acceso a los servicios del Estado y del trato que brinda este, en tanto que este provea los recursos y servicios para una adecuada existencia, salvaguarde sus derechos y les brinde condiciones para una mejor calidad devida.

En cuanto a la valoración de los principales líderes políticos del país, los resultados de estas variables son interesante dado su significado para el modelo. Por ejemplo, respecto a la valoración de Pedro Pablo Kuczynski como líder político, el modelo no la toma como significativa para explicarlo. No obstante, el modelo sí considera como significativas las valoraciones de los liderazgos de las principales rivales políticas del candidato: Keiko Fujimori y Verónica Mendoza -en relación directa-, se entiende que cuanto mayor sean valorados sus liderazgos por los votantes de PPK, mayor será la satisfacción que sientan estos con el sistema. Esto tiene como base que en un sistema democrático como tal, la tolerancia hacia otras tendencias políticas es importante, dado que dicha tolerancia es la base de todo el sistema político y, asimismo, es lo que permite la alternancia del poder entre diferentes opciones, dando oportunidad a todas las tendencias ideológicas en el gobierno, lo que reforzaría la idea de la participación de partidos políticos de diferentes corte ideológico mas siempre que respeten el sistema democrático y lo crean legítimo.

Finalmente, el modelo estima como variables significativas el grado de acuerdo que tienen los votantes de PPK sobre la aseveración "como la política es compleja, muchas veces los ciudadanos no logramos entenderla", se puede entender que mientras la percepción de esta -en otros términos, los ciudadanos perciben que la política es muy compleja y les es difícil entenderla- sea menor, habrá una mayor satisfacción con el sistema democrático, dado que podrán entender el quehacer político de la sociedad y del 
Estado y, por lo tanto, se formarían un criterio sobre esta, discutirla y, en consecuencia, de una opinión.

\subsection{Factores que influyen en la satisfacción con la democracia de los votantes de Fuerza Popular (FP) y a su candidata Keiko Fujimori (losperdedores)}

El siguiente modelo trabajado encontró las relacionales causales entre las la satisfacción con la democracia -variable dependiente- de los ciudadanos que recuerdan haber votado en segunda vuelta - ballotage- por el partido político Fuerza (FP) y su candidata Keiko Fujimori -filtro- y los factores que la determinan o la influyen -variables independientes.

Respecto a las variables causales-explicativas del modelo, la más importante de todas tiene que ver con el apoyo al sistema democrático, lo que determina que mientras más se apoye el sistema democrático es más probable que los votantes de Fuerza Popular se sientan satisfechos con el sistema político. Esta relación causal es importante para una agrupación política como Fuerza popular, ya que con esto apoyaría la tesis de una superación de la estigmatización que tiene esta por una parte de la población, dada su vinculación con el régimen de gobierno de la década de 1990 -denominada como la época del autoritarismo de Alberto Fujimori, padre de la candidata-. Asimismo, teniéndose en cuanto este precedente, existe aún cierto miedo por parte de una gran parte de la población a un eventual gobierno fujimorista, debido principalmente a esta idea de la herencia autoritaria y, dada la evidencia de su trabajo en los últimos tres gobiernos, en cuanto a no desligarse de dicho pasado del todo. No obstante, a pesar de esto hoy en día los votantes de Fuerza Popular estiman que el apoyo al sistema democrático es muy significativo para sentirse satisfechos con este, debido a que dada las reglas del juego institucionales y las eventuales correlaciones de fuerza y de poder que se puedan dar en el presente y futuro, esta agrupación podría llegar nuevamente al poder. En la necesidad de reforzar esta idea, también se puede observar la relación de la variable "respeto al estado de derecho", la cual se relaciona directamente con la satisfacción con la democracia y también tiene un peso de ocurrencia estadístico bastante relevante. De este modo, gracias a la significatividad que tienen en el modelo explicativo, podríamos decir que hoy en día los votantes de Fuerza Popular entienden la importancia de apoyar a la democracia y de enmarcar su actividades políticas dentro de los márgenes de la legalidad, dado que en el pasado no lo hicieron y trajo como consecuencia una estigmatización de este agrupación política que se evidenció en el veto político de sus principales líderes, en cuanto a no poder ejercer cargos políticas por 10 años - los procesos de inhabilitación temporal para el ejercicio de la función pública.

Asimismo dentro de las variables sociodemográficas que acoge el modelo, estas son el sexo (género) y la situación laboral -y ambas en forma directa-. Respecto a la primera, se interpreta que entre los votantes de Fuerza Popular, los varones son los que se sienten más satisfechos con el sistema que las mujeres. Esta relación también se puede evidenciar en los resultados descriptivos del perfil del votante de Fuerza Popular, tanto de los que están más satisfechos con la democracia -en su mayoría varones- y los que no -en su mayoría mujeres. En cuanto a la relación con la situación laboral, se colige que mientras los votantes de Fuerza Popular se encuentren en una mejor situación laboral, mayor será la satisfacción que sientan con el sistema político democrático. Asimismo, se observa que la mayoría de los votantes de Fuerza Popular contestaron ser independienteslaboralmente. 
Respecto a la confianza en las instituciones del estado, las que acoge el sistema son el Gobierno Nacional, el Congreso de la República, el Jurado Nacional de Elecciones (JNE) y la Defensoría del Pueblo. En cuanto a la confianza al Gobierno Nacional y al Jurado Nacional de Elecciones, ambas en relación directa y significativa, se puede interpretar que, para el modelo dado, a mayor confianza que se tenga en el Gobierno Nacional, en cuanto a su desempeño, y en el adecuado funcionamiento del Jurado Nacional de Elecciones, mayor será la satisfacción con el sistema democrático -en términos de funcionalidad de estas en el sistema, dado que el primero representa al ejecutivo, en tanto que es quien tomará las riendas del gobierno, dirigirá las carteras ministeriales, será el quien ejecutará las leyes, y distribuirá y redistribuirá el presupuesto nacional, en tanto tiene a la cabeza al Presidente, Presidente de Consejo de Ministros y al Ministro de Economía y Finanzas- y, el segundo, al sistema electoral en su conjunto -dado que esta se asocia a los procesos electoral en calidad de ser el tribunal electoral y quien determina las reglas del juego del mismo, en primera y última instancia-.

Respecto a la confianza en el Congreso de la República y la Defensoría del Pueblo, se evidencia que mientras la confianza en estas sea menor, mayor será el grado de satisfacción con el sistema político. Este dato resulta inesperado, en tanto que la Presidencia del Congreso Nacional recae en el partido con mayoría relativa, es decir, en el partido por el cual los votantes, en su mayoría, apostaron por sus candidatos en las urnas; no obstante, esta relación inversa podría entenderse en tanto a la acción de esta institución sobre el desarrollo de la gobernabilidad en estos meses post toma de mando por el nuevo gobierno, el cual se enfrenta con la fuerte oposición del Congreso Nacional al ser un grupo parlamentario en minoría. En cuanto a la confianza en la Defensoría del Pueblo, la relación con la variable satisfacción con sistema democrático es inversa, lo que podría interpretarse como una respuesta contestataria, dado el grado identificación de los encuestados con la propuesta fujimoristas la cual apoyan y, en consecuencia, asumen como legítimas las decisiones tomadas de su líder Alberto Fujimori, las cuales muchas veces han sido materia de investigación por dicha institución, así como también ha descalificado algunos hechos en sus pronunciamientos e informes.

Respecto a las variables estructurales relevantes para el modelo establecido, se evidencia una relación directa entre la valoración de la situación económica actual del país - en términos de una buena gestión de la economía y de la implementación de políticas económicas que amplíen la distribución de los recursos homogéneamente y que permita la obtención de recursos a la gran mayoría de ciudadanos -crecimiento económico equitativo, estable y con desarrollo social.

Respecto a la percepción de la corrupción en el sistema, por un lado se tiene la variable de la percepción de cuántos políticos están inmersos en actos de corrupción, la cual se entiende que más políticos estén inmersos en actos de corrupción -pocos o casi ninguno-, mayor será la satisfacción con el sistema democrático para los que admiten haber votado por FP en las últimas elecciones. No obstante, por el lado de la percepción de cuántos funcionarios públicos están implicados en actos de corrupción, se interpreta que mientras menos funcionarios estén implicados en casos de este tipo, mayor será la satisfacción con el sistema. 
Respecto a la aprobación de los principales funcionarios públicos del estado peruano y de los principales representantes de la oposición, tanto en el Congreso Nacional como del ejecutivo; la variable más relevante dentro de la aprobación de la gestión los funcionarios públicos es la aprobación de la gestión del Presidente del Consejo de Ministros Fernando Zavala, del cual se entiende que mientras mejor sea su desempeño mayor será la sensación de satisfacción con el sistema democrático. Además, respecto a la evaluación de la gestión de los líderes de oposición en el gobierno, aquí la variable más significativa está ligada a la aprobación de la gestión de Verónica Mendoza en la oposición, cuya relación con esta variable es que mientras menos se apruebe la gestión de Mendoza en la oposición, mayor será la satisfacción que estos sientan con el sistema. Esto se podría entender en tanto que Mendoza es la representante de las fuerzas de izquierda en el Perú, la cual es rechazada por una amplia mayoría fujimorista, debido a la inestabilidad política que provocó la izquierda política radical y violenta durante la década de 1980 hasta inicios del 1990, por lo cual también legitiman el gobierno de Fujimori padre al ser el principal líder político y de gobierno que luchó para controlar el avance terrorista. La izquierda peruana perdió peso en la lucha contra el terrorismo al no hacer un deslinde oportuno entre esta y las acciones violentas de los grupos de extrema izquierda en elpaís.

En cuanto a la valoración de los principales líderes políticos del país, los resultados de éstas son interesantes dado su significado para el modelo. Por ejemplo, respecto a la valoración de Pedro Pablo Kuczynski como líder político, se observa que mientras menos sea valorado este como tal, mayor será la probabilidad de sentir una mayor satisfacción con el sistema democrático. No obstante, respecto a la valoración del liderazgo de Verónica Mendoza,se entiende que cuanto mayor sea valorado su liderazgo, mayor será la satisfacción con el sistema. Esto se interpretaría en tanto a la rivalidad política de sus opositores y la posición ideológica de los mismos, dado que Kuczynski estaría dentro de un espectro ideológico similar al de Fujimori y el espacio electoral que tiene este es compartido con el de Fujimori y, por lo tanto, este candidato fue su principal rival electoral en las últimas elecciones y fue con quien paso a la segunda vuelta de esta; mientras que en el caso de Verónica Mendoza, los espacios políticos de influencia y de mercado electoral están marcadamente distanciados, lo cual no la tuvo como una verdadera rival en las elecciones - esto dado que Mendoza es un lideresa de izquierda y el mercado electoral de esta no comulgaba con la propuesta política de Fuerza Popular, por lo que esta representa, para el votante fujimorista promedio y en términos de radicalismo político, inseguridad política- económica-social y jurídica, entre otros.

Finalmente, el modelo estima como variables significativas el grado de acuerdo que tienen los votantes de FP ante la aseveración "entiendo bastante bien las cuestiones políticas más importantes del país" y el grado de acuerdo sobre "los políticos no se preocupan por las demandas ciudadanas". Respecto a la primera, su relación con la satisfacción con la democracia en tanto que mientras menor sea el acuerdo en que ellos entienden bastante sobre las cuestiones políticas más importante, mayor será la satisfacción con el sistema político; es decir, que mientras menos entiendan los votantes de FP sobre las cuestiones más importante de la política, más satisfechos estarán con el sistema. Asimismo, respecto a la segunda aseveración, se interpretaría que mientras el grado de acuerdo sobre esta sea menor, mayor será la satisfacción con el sistema; es decir, mientras más los políticos se preocupen por las demandas de los ciudadanos, mayor será la satisfacción que tengan estos con el sistemapolítico. 


\section{CONCLUSIONES}

Respecto sobre los factores que influyen en la satisfacción con el sistema para los que votaron por Kuczynski, el más importante de todos es el buen desempeño que tenga el ministro de Economía y Finanzas, lo cual evidencia la gran importancia para los simpatizantes y electores de Peruanos por el Kambio el funcionamiento de la economía.

Asimismo dentro de las variables sociodemográficas, las únicas que acoge el modelo son la situación laboral y el nivel de ingresos, las cuales se relaciona directamente con la sensación de satisfacción con el sistema político democrático en tanto que mientras mejor sea la percepción de estar en una situación laboral que les permita, como mínimo, tener ingresos que satisfagan mis necesidades se sentirán satisfechos con el sistema -en este caso el grupo mayoritario de los encuestados respondieron ser independientes o emprendedores Otras variable significativa tiene que ver con la auto-ubicación étnica de los votantes a PPK, se entiende que los votantes de origen étnico quechua son los que menos satisfechos se sienten con la democracia. La interpretación en este caso podría darse dada la existencia de una marcada diferenciación entre los grupos étnicos en el Perú y su adecuado acceso a los servicios del Estado y del trato que este les brinda - problema de inclusión de los grupos menos favorecidos históricamente hablando.

Asimismo, para los electores de PPK la Policía Nacional y el Jurado Nacional de Elecciones (JNE) son las instituciones más importantes y la confianza en estas determina el nivel de satisfacción que sientan con el sistema, lo que se interpreta como la confianza hacia el sistema electoral, como símbolo de la legalidad del sistema electoral, y el sistema de seguridad interna del país y su funcionamiento como primordial en cuanto al funcionamiento del sistema político.

Como se ha visto en los otros casos, tanto de los satisfechos como de los insatisfechos con el sistema, la variable económica y el adecuado funcionamiento de las instituciones de gobiernos ligadas a esta son de vital importancia para la satisfacción con el sistema democrático. Por ello, mientras mejor vaya la economía del país y mientras haya un optimismo sobre la posibilidad de mejora de la situación económica personal y familiar respecto a cómo era un año atrás, mayor será la satisfacción que tenga con el sistema democráticos los votantes de Pedro Pablo Kuczynski.

Sobre la gestión de los líderes de oposición en el gobierno, los electores de PPK consideran que la satisfacción que sientan sobre el sistema está relacionada inversamente a la aprobación de la gestión de las lideresas Verónica Mendoza y de Keiko Fujimori en la oposición. En cuanto a la valoración de los principales líderes políticos del país, el modelo sí considera como significativas las valoraciones del liderazgo de las principales rivales políticas del candidato: Keiko Fujimori y Verónica Mendoza -en relación directa-, mientras más valoren a estas lideresas los votantes de PPK, mayor será la satisfacción que sientan estos con el sistema, dado que legitima los liderazgo como fuerzas de oposición y la participación política de las fuerzas opositoras también es parte de la consolidación del sistema político -tolerancia es la base de todo el sistema político, permite la alternancia del poder entre diferentes opciones, dando oportunidad a todas las tendencias ideológicas en el gobierno, lo que reforzaría la idea de la participación de partidos políticos de diferentes corte ideológico. 
Finalmente, los votantes de PPK perciben que si bien la política es muy compleja, pero si el grueso de la ciudadanía logra entenderla, más satisfechos se sentirán con el sistema democrático, dado que podrán entender el quehacer político de la sociedad y el Estado y, por lo tanto, se formarían un criterio sobre esta y podrán discutirla y hacerse de unaopinión.

En cuanto a los factores que influyen en la satisfacción que sienten por la democracia los votantes de Fuerza Popular, el factor más importante de todas tiene que ver con el apoyo al sistema democrático, ya que estos al apoyar más el sistema democrático se sentirán satisfechos con este. Esta relación es importante ya que hasta hace un tiempo atrás, continuaban siendo estigmatizados como autoritarios, resultado de la vinculación con el régimen de gobierno de la década de 1990 liderado por Alberto Fujimori, padre de la candidata. Sin embargo, también es importante resaltar que existe aún un sector del fujimorismo que no acepta dicha denominación al gobierno de esta etapa.

Otra variable influyente en esta relacionada con el sexo y la situación laboral, en cuanto que son los votantes varones quienes se sienten más satisfechos con el sistema en comparación a las mujeres votantes de FP -esto también se evidencia en los resultados del análisis descriptivo del perfil del votante de Fuerza Popular. En cuanto a la relación de la situación laboral, mientras los votantes de Fuerza Popular se encuentren en unamejor situación laboral, más satisfechos estarán con el sistema frente a los queno.

La confianza que tengan sobre el Gobierno Nacional, el Congreso de la República, el Jurado Nacional de Elecciones (JNE) y la Defensoría del Pueblo determinará su satisfacción con el sistema democrático. Las que se relacionan de forma directa con la satisfacción son la confianza al Gobierno Nacional y al Jurado Nacional de Elecciones, ya que a mayor confianza que se tenga en estás, mayor será la satisfacción de los votantes de FP con el sistema democrático -en otros términos, confían el en sistema de gobierno y en el sistema electoral, los cuales sustentan de manera directa el apoyo al sistema y la calidad de su funcionamiento repercutirá significativamente en la satisfacción con este.

No obstante, en cuanto a la Confianza en el Congreso de la República y la Defensoría del Pueblo, mientras menor sea esta, mayor será el nivel de satisfacción con el sistema político, lo cual resulta contradictorio en el caso del Congreso de la República, dado que es FP quien tuvo la Presidencia de la mesa directiva del Congreso Nacional en legislativos por ser el partido político de mayoría -relativa-, es decir, en el partido por el cual estos votantes apostaron en las urnas para el congreso -aunque esto también se evidenciaba en el análisis descriptivo-; no obstante, en cuanto a la Defensoría del Pueblo, esto podría entenderse yaque esta institución ha sido una de las que más ha cuestionado las políticas adoptadas en el gobierno de Alberto Fujimori, las cuales muchas veces han sido materia de investigación por dicha institución, y también ha descalificado dichos hechos en pronunciamientos e informes oficiales.

Asimismo, y como en todos los modelos analizados, la parte económica tiene también una fuerte influencia en la satisfacción con la democracia para los votantes de FP, ya que es importante para estos que la situación económica actual del país vaya como hasta ahora 
o mejore para que sigan satisfechos con el sistema democrático -en términos de una buena gestión de la economía y la distribución de los recursos sea homogénea permitiendo la a la gran mayoría de ciudadanos vivir mejor.

No obstante, un dato muy significativo respecto a la cantidad de políticos y funcionarios públicos implicados en casos de corrupción, mientras más políticos estén inmersos en actos de corrupción - pocos o casi ninguno-, mayor será la satisfacción con el sistema democrático para los que admiten haber votado por FP en las últimas elecciones. No obstante, por el lado de la percepción de cuántos funcionarios públicos están implicados en actos de corrupción, se interpreta que mientras menos sean funcionarios estén implicados en casos de este tipo, mayor será la satisfacción con el sistema. Por lo tanto, se evidencia un cierto grado de tolerancia por los votantes de FP hacia actos de corrupción de los políticos no funcionarios. No obstante, es importante también resaltar la no tolerancia de la corrupción en los funcionarios públicos, debido a que estos tienen el manejo de los recursos públicos y tienen la capacidad de decisión sobre la política de unpaís.

Por otro lado, en cuanto a la aprobación de la gestión los funcionarios públicos, el más importante de todos es el Presidente del Consejo de Ministros, en este caso Fernando Zavala y esta se relaciona de forma directa con la satisfacción que sientan los votantes de FP con la democracia, es decir mientras a mejor sea su desempeño mayor será la sensación de satisfacción con el sistema democrático, lo cual evidenciaría la necesidad de un buen gobierno del ejecutivo para la satisfacción con el sistema. No obstante, lo mismo no pasa con la aprobación de la gestión de Verónica Mendoza en la oposición, ya que los votantes de FP consideran que mientras menor sea la aprobación de la gestión de esta, mayor será la satisfacción que estos sientan con el sistema. Esto se podría entender en tanto que Mendoza es la representante de las fuerzas de izquierda en el Perú, la cual es rechazada por una amplia mayoría fujimorista. La izquierda peruana perdió peso y hasta algo de legitimidad en el escenario político peruano al no hacer un deslinde oportuno entre esta y las acciones violentas de los grupos de extrema izquierda en elpaís.

En cuanto a la valoración de Pedro Pablo Kuczynski como líder político, este es menos valorado por los votantes de FP y, por lo tanto, esto aumentará el nivel de satisfacción con la democracia que estos tengan. No obstante, esto no sucede en cuanto a la valoración del liderazgo de Verónica Mendoza, se entiende que cuanto mayor sea valorado su liderazgo, mayor será la satisfacción con el sistema. La rivalidad política con Kuczynski estaría dentro de un espectro ideológico similar al de Fujimori y el espacio electoral que tiene este es compartido y, por lo tanto, este candidato fue su principal rival electoral; mientras que en el caso de Verónica Mendoza, los espacios políticos de influencia y de mercado electoral están marcadamente distanciados y, finalmente, los votantes de FP sienten más satisfacción con el sistema democrático mientras entiendan menos las cuestiones políticas más importante del país y mientras los políticos se preocupen por las demandas ciudadanas. 


\section{REFERENCIAS BIBLIOGRÁFICAS}

ALISTER, C., CEA, C., Y GUERRERO, A., (Agosto 2015). Democracia en América Latina ¿Qué factores influyen en la satisfacción y apoyo a la democracia? FRONTERAS, Revista de Ciencias Sociales y Humanidades. Recuperado de http://publicacionescienciassociales.ufro.cl/index.php/fronteras/article/view/445

ANDUIZA, E., CRESPO, I. Y MÉNDEZ, M. (1999). Metodología de la Ciencia Política. Lima: Fondo de Desarrollo Editorial.

CAMPBELL, AUGUST, PHILIP CONVERSE, WILLIAM MILLER Y DONALD STOKES (1960). The American Voter. New York: Miller.

DIAMOND, L., MORLINO, L., (2005). Assesing the Quality of Democracy. Baltimore: John Hopkins University Press.

DOWNS, A. (1957). An Economic Theory of Democracy. Nueva York: Harper Collins Publishers.

HERNÁNDEZ, R., FERNÁNDEZ, R. BAPTISTA, P. (2014). Metodología de la Investigación Cientifica. Edamsa: México. Held, D., (1996). Modelos de Democracia. Segunda reimpresión. España: Alianza UniversalEditorial.

HELD, D. (1996). Modelos de Democracia. Madrid: Alianza Editorial.

INSTITUTO DE OPINIÓN PÚBLICA DE LA PONTIFICIA UNIVERSIDAD CATÓLICA DEL PERÚ. Encuesta de Opinión pública preelectoral 2011 y base de datos. Recuperada de: http://iop- data.pucp.edu.pe/busqueda/encuesta/70?

INSTITUTO DE OPINIÓN PÚBLICA DE LA PONTIFICIA UNIVERSIDAD CATÓLICA DEL PERÚ. Encuesta de Opinión pública preelectoral 2016 y base de datos. Recuperada de: http://iop- data.pucp.edu.pe/busqueda/encuesta/120?

INSTITUTO DE OPINIÓN PÚBLICA DE LA PONTIFICIA UNIVERSIDAD CATÓLICA DEL PERÚ. Encuesta de Opinión pública postelectoral 2016 y base de datos. Recuperada de: http://iop- data.pucp.edu.pe/busqueda/encuesta/121?

LAZO, S., (2015). Comportamiento electoral en el Perú: Un análisis del rol de las variables sociodemográficas y sociodemográficas y socioeconómicas en las elecciones presidenciales en primera vuelta de 2006 y 2011. Revista de Revista de Ciencia Política y Gobierno, 2(3), 51-80. 
LEVINE, D. Y MOLINA, J. E., (Editores) (2011). The Quality of Democracy in Latin Americ. London: Lynne Rienner Publishers Inc.

MAHEIM, J. Y RICH, R., (2000). Análisis político empírico y Métodos de investigación en ciencia política. Madrid: Alianza Editorial.

MELÉNDEZ, C. (2012). Partidos inesperados. La institucionalización del sistema de partidos en un escenario de post colapso partidario. Perú 2001-2011. Análisis y Debate. Fundación Friedich Ebert Stiftung. Recuperado de: http://www.fes.org.pe/descargasFES/Partidos\%20inesperados\%20C.\%20Melendez.pdf

MELÉNDEZ, C. (2019). El Mal Menor. Vínculos políticos en el Perú posterior al colapso del sistema de partidos. Recuperado de https://books.google.com.pe/ books?id=1LKIDwAAQBAJ\&pg=PT4\&source $=$ gbs_toc_ $\quad$ r\&cad $=3 \# \mathrm{v}=$ onepage \&q\&f=false

MONTECINOS, E., (2007). Análisis del comportamiento electoral: De la elección racional a la teoría de redes. Revistas de Ciencias Sociales (Ve), 13(1), 9-22.

PARDINAS, F. (2005). Metodología y Técnicas de investigación en Ciencias Sociales. Madrid: Siglo XXI.

SULMONT, D. (2009). Una revisión sobre la literatura de los estudios sobre el comportamiento electoral en el Perú. Doctorado de Ciencia Política, pp. 1-20 (4,469) 